\title{
LARGE EDDY SIMULATION OF TURBULENT PARTICLE-LADEN CHANNEL FLOW CONSIDERING TURBULENCE MODULATION BY PARTICLES
}

\begin{abstract}
Jaroslav VOLAVY ${ }^{\star}$, Matej FORMAN ${ }^{\star *}$, Miroslav JICHA ${ }^{\star \star \star}$
Abstract: Large Eddy Simulation of vertical turbulent channel flow laden with particles are performed. The number of particles is chosen very large and the volume fraction of particles is high enough for consideration of two-way coupling. This means that the particles are influenced by fluid and vice versa. The inter-particle collisions are neglected. The Euler-Lagrange method is adopted, which means that the fluid is considered to be continuum (Euler approach) and for each individual particle is solved Lagrangian equation of motion. Particles are considered to be spherical. The simulations are performed for different volume fractions of particles in the channel. The results are compared to the single-phase channel flow in order to investigate the effect of the particles on the turbulence statistics of the carrier phase.
\end{abstract}

\section{INTRODUCTION}

One of the most interesting problem of fluid dynamics is the solution of turbulent flow with the presence of disperse phase in the mainstream. This type of flow can be found in many industrial applications, eg in the chemical industry, coal-fired power plants in the transportation of coal dust to the boiler, in the prediction of air pollution and many others. If we want to determine with sufficient precision movement of disperse phase (particles), it is very important to resolve the flow and turbulence of the carrier phase, because this affects transportation and deposition of particles most. In cases where the concentration of particles in the flow is not great (volume fraction of disperssed and carrier phase $\alpha_{p}<10^{-6}$ ), we only consider the so-called one-way coupling [9]. This means that the fluid affects the particles and it is not affected by the presence of the particles ("stream does not know about particles"). Whenever the volume fraction exceeds the value of $\alpha_{p}>10^{-6}$, then the presence of the particles could not be neglected and it is necessary to use two-way coupling (fluid flow affects the particles and vice versa). In this case the turbulence is modulated by particles. The question arises, how turbulence is modulated by particles and how this modulation should be included in computer code solving the two-phase flow.

\section{GoVERNING EQUATIONS}

The system of carrier phase (liquid) and dispersed phase (particles) is described in this work using Euler-Lagrange approach. This means that the liquid is considered to be continuum and its motion is described by the Euler equation of motion. The particles are considered as mass points and for their simulation is used Lagrangian approach. For each particle is assembled equations of motions based on the second Newton's law.

\footnotetext{
* Jaroslav Volavy, Faculty of Mechanical Engineering, Brno University of Technology, Technická 2896/2, 616 69 Brno, Czech Republic, jaroslav.volavy@gmail.com

${ }^{* *}$ Matej Forman, Faculty of Mechanical Engineering, Brno University of Technology, Technická 2896/2, 61669 Brno, Czech Republic, forman@fme.vutbr.cz

${ }^{* * *}$ Miroslav Jicha, Faculty of Mechanical Engineering, Brno University of Technology, Technická 2896/2, 616 69 Brno, Czech Republic, jicha@fme.vutbr.cz
} 


\subsection{LIQUID PHASE}

For the solution of the fluid flow in this article was chosen Large Eddy Simulation. The main idea of Large Eddy Simulation is to separate large scales (grid-scales) from small scales (subgrid-scales) to lower computational cost. The subgrid scales are modelled using subgrid model. The scale separation is done by applying filter operator on Navier-Stokes equation. If we apply the filter operator on NavierStokes equations we obtain filtered Navier-Stokes equations:

$$
\frac{\partial \bar{u}_{i}}{\partial t}+\frac{\partial}{\partial x_{j}}\left(\bar{u}_{i} \bar{u}_{j}\right)=-\frac{1}{\rho} \frac{\partial \bar{p}}{\partial x_{i}}+\nu \frac{\partial^{2} \bar{u}_{i}}{\partial x_{k} \partial x_{k}}-\frac{\partial \tau_{i j}}{\partial x_{j}}-f_{i},
$$

where $f_{i}$ is coupling force which takes into account the presence of the particles. This force is defined as:

$$
f_{i}=\sum_{n=1}^{N} f_{n, i}
$$

$f_{n, i}$ is the $i$-th acting on the $n$-th particle. Force $f_{n, i}$ is equal to the rand hand side of 5 .

For evaluation of subgrid stress tensor $\tau_{i j}$ is used subgrid kinematic energy model:

$$
\tau_{i j}=-2 \nu_{k} \bar{S}_{i j}+\frac{2}{3} k_{s g s} \delta_{i j},
$$

where $\bar{S}_{i j}=\partial \bar{u}_{i} / \partial x_{j}+\partial \bar{u}_{j} / \partial x_{i}$ and $k_{s g s}$ is obtained from equation:

$$
\frac{\partial k_{s g s}}{\partial t}+\bar{u}_{i} \frac{\partial k_{s g s}}{\partial x_{i}}=-\tau_{i j} \frac{\partial \bar{u}_{i}}{\partial x_{j}}-C_{c} \frac{k_{s g s}^{3 / 2}}{\Delta}+\frac{\partial}{\partial x_{j}}\left(\frac{\nu_{k}}{\sigma_{k}} \frac{\partial k_{s g s}}{\partial x_{i}}\right) .
$$

The constants in equation (4) are set as follows: $C_{k}=0.05, C_{c}=1.0 \mathrm{a} \sigma_{k}=1.0$.

\subsection{SOLID PHASE}

The motion of particles is described by Lagrangian equations of motion for each particle. The only force considered here is drag force. Because of high concentration of particles we consider the influence of particles on the fluid. The equation of motion for particle is ${ }^{2}$ :

$$
\frac{\mathbf{d v}_{j}}{\mathrm{~d} t}=\frac{\mathbf{u}\left(\mathbf{x}_{j}, t\right)-\mathbf{v}_{j}}{\tau_{p}}\left(1+0.15 \operatorname{Re}_{p}^{0,687}\right),
$$

where $\mathbf{v}_{j}$ is velocity of $\mathbf{j}$-th particle, $\mathbf{u}\left(\mathbf{x}_{j}, t\right)$ is velocity of fluid on particle position $\mathbf{x}_{j}, \tau_{p}=\rho_{p} d_{p}^{2} /\left(18 \rho_{f} \nu\right)$ is particle relaxation time. The standard drag correlation is applied.

\section{Test CASE}

The simulation of particle-laden turbulent channel flow was performed. The simulated flow field is a fully developed turbulent flow between two parallel walls. Hence, the flow is homogeneous both in the streamwise and spanwise directions and the statistics are dependent only upon the distance from the wall.

The dimensions of the channel are $4 \mathrm{~m}$ in streamwise direction and $2 \mathrm{~m}$ in spanwise and wall-normal direction. Periodic boundary condition is prescribed in the streamwise and spanwise direction. For $y=0$ and $y=2 m$ is adjusted wall boundary condition. Computational grid consist of $40 \times 50 \times 30$ cells. The spacing both in streamwise and spanwise direction is uniform. The mesh becomes finer towards the wall in order to capture turbulence generation in the near-wall region. On the wall is satisfied condition $y^{+}=y u_{\tau} / \nu \approx 1$. The grid spacing is in the streamwise and spanwise direction $\Delta x^{+}=28$ and $\Delta z^{+}=13$. The simulations were performed for high Reynolds number $\left(R e_{\tau}=400\right)$.

The volume fractions of the particles contained in the flow are $\alpha_{p}=2 \cdot 10^{-6}$ and $\alpha_{p}=5 \cdot 10^{-6}$. The concentration was chosen sufficently high in order to get effect on the liquid phase. Turbulenc should be modulated by particles. Particles are supposed to be spheres. The diameter of the particles are governed by Rosin-Rammler statistical distribution. The mean value of diameter is $0.0001 \mathrm{~m}$. On the begining of the simulation are uniformly distributed in the channel and the velocity of the particles are same as the velocity of the fluid. The initial configuration of the simulation is in the figure 1 . The size of the particles in the figure was enlarged in order to be seen. 

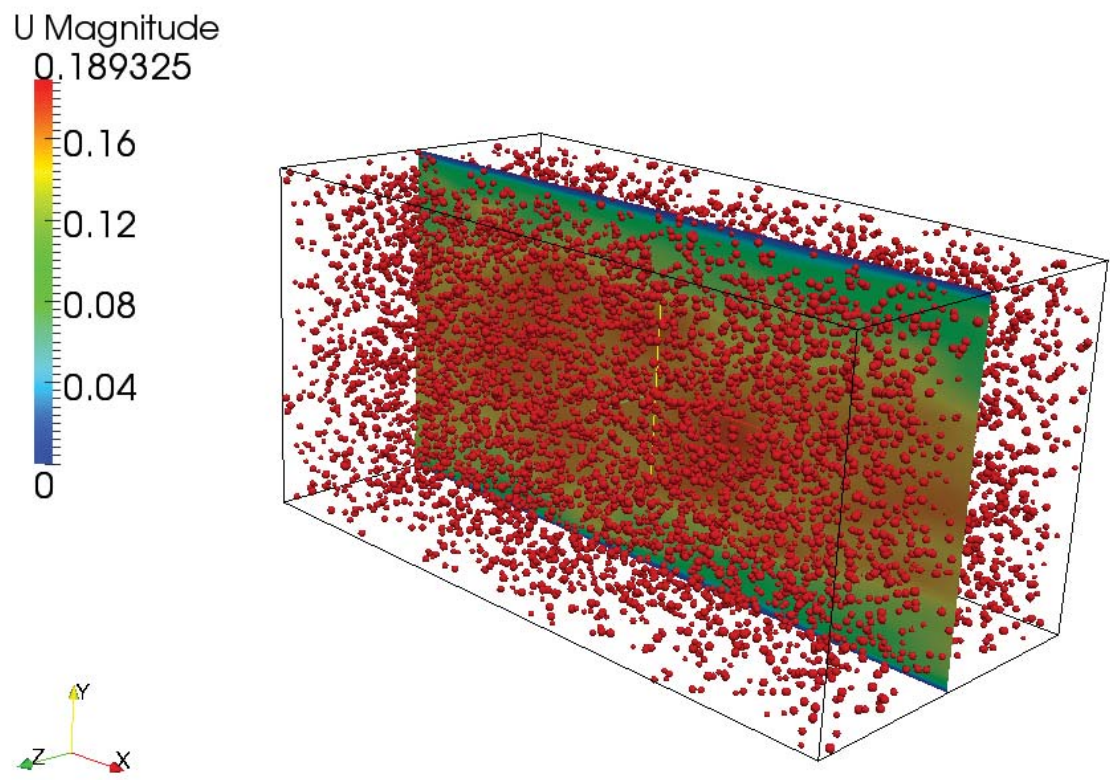

Figure 1: Initial configuration of the simulations

\section{Results}

In this section are introduced results of simulations of particle-laden channel flow. First was done simulation without particles and the data from this simualtion was used for comparision particle-laden flows in order to investigate the effect of the particles to the turbulence. The simulations with particles was performed for various volume fractions of particles: $\alpha=2 \cdot 10^{-6}$ and $\alpha=5 \cdot 10^{-6}$.

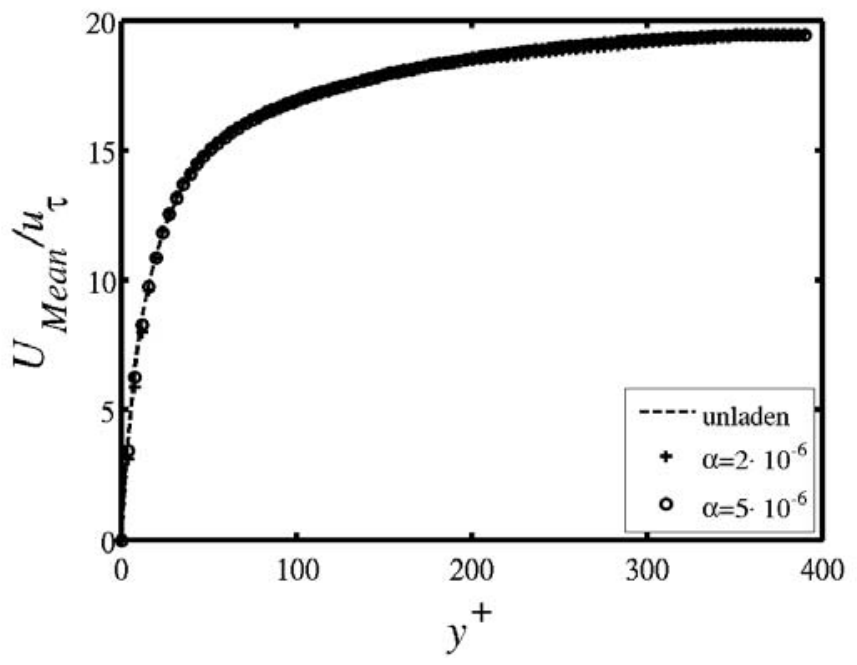

Figure 2: Velocity profile across channel

In the figure 2 is depicted velocity profile accros the channel. The profile was found to be symmetric 
across the channel centerline to within $0.5 \%$. It could be noticed that the mean velocity profile in the channel is not affected by presence of the particles. From view of mean velocity could particles be regarded as neglectable for both volume fractions of particles.

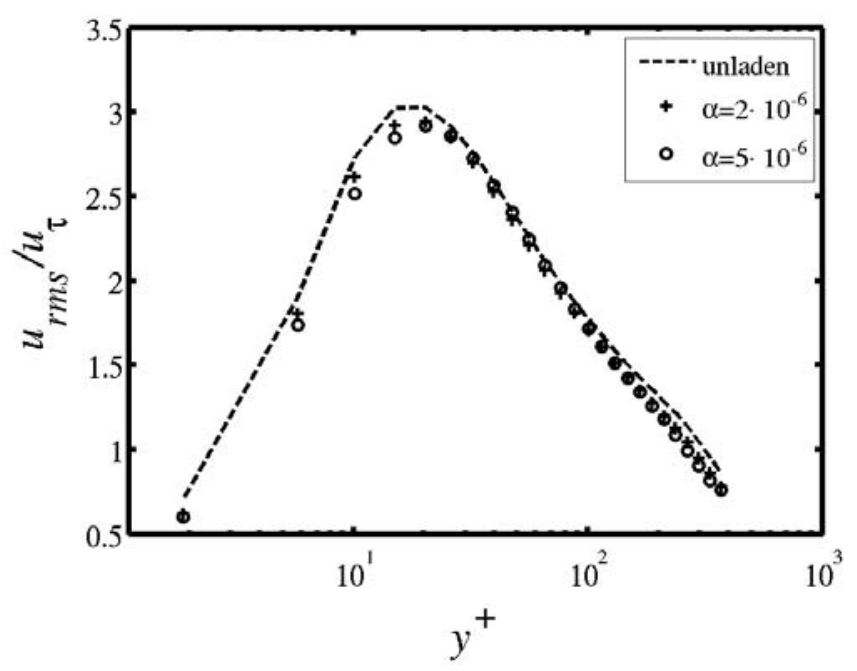

Figure 3: Streamwise fluid velocity fluctuation

Streamwise fluid velocity fluctuations are in figure 3 . This figure implies that the influence of the particles on the fluid turbulence has indispenasable effect. In the near-wall region is turbulence damped more than in the rest of the channel. In region $y^{+} \approx 70$ seems turbulence fluctuation untouched for both concentration of particles. The turbulence is attenuated because the motion of the particles drains energy from the mainstream. Particles used in these simulations are small so the wake behind particels is also very small and therefore has no significant effect. If the wake would be sufficiently great the turbulence would be generated by particles.

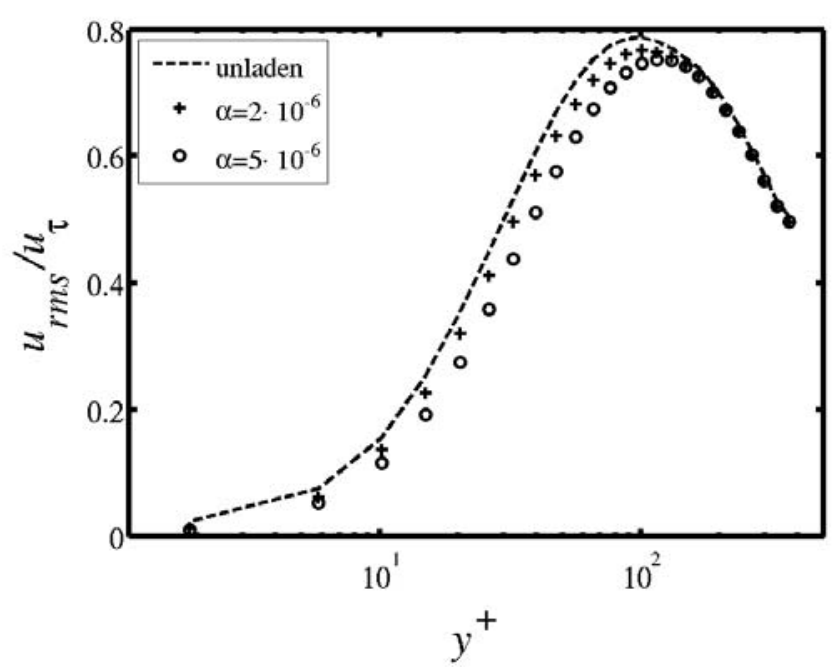

Figure 4: Wall-normal fluid velocity fluctuation

The wall-normall velocity fluctuations are affected in the same manner as streamwise fluctuations, see figure 4. The role of concentration of particles in turbulence modulation is obvious from the figures. Higher volume fraction of particles leads to the more significant turbulence attenuation. 


\section{Conclusion}

The simulations of particle-laden channel flow were performed. For the description of the system fluidparticle was used Euler-Lagrange approach. The simulation was done using two-way coupling. This means, that the influence of particles on the fluid was not neglected but was taken into account during simulation. The coupling between liguid and dispersed phase was achieved by adding special term to the momentum equation of motion. This term equals to the sum of forces acting on every particle. The concentration of the particles was chosen high enough to observe turbulence modulation.

For the solution of the fluid motion was used Large Eddy Simulation method. The motion of the particles was described by Lagrangian equations of motion. These equations was solved using second order Euler scheme. The simulations were done for two different concentrations of particels in the channel. It has shown, that the presence of the particels tends to attenuate turbulence. The intensity of the attenuation is not constant accros the channel. In the near-wall region is the turbulence more damped than in the rest of the channel.

\section{ACKNOWLEDGMENT}

The support of grant GA ČR 101/08/0096 as well as the project FSI-J-10-7 is gratefully acknowledged.

\section{REFERENCES}

[1] Boivin, M., Simonin, O., Squires, K. D.: On the prediction of gas-solid flows with two-way coupling using large eddy simulation, Phys. Fluids, Vol. 12, No. 8, 2000

[2] Elghobashi, S., Truesdell, G. C.: On the two-way interaction between homogeneous turbulence and dispersed solid particles. I: Turbulence modification, Phys. Fluids A, Vol. 5, No. 7, 1993

[3] Ferrante, A., Elgobashi, S.: On the physical mechanism of two-way coupling in particle-laden isotropic turbulence, Phys. Fluids, Vol. 15, No. 2, 2003

[4] García, J.: Study of the turbulence modulation in particle-laden flow using LES, Annual Research briefs, Centre for turbulence Research, 2001

[5] Gore, R. A., Crowe, C. T.:Modulation of Turbulence by a Dispersed Phase, Trans. ASME I: J. Fluid Eng. 113, 1991

[6] Kulick, J. D., Fessler, J. R.,Eaton, J. K.: Particle response and turbulence modification in fully developed channel flow, J. Fluid Mech., vol. 277, 1994

[7] Mandø, M., Lightstone, M. F., Rosendahl, L., Yin, C., Sørensen, H.: Turbulence modulation in dilute particle-laden flow, Int. J. Heat and Fluid Flow 30, 2009

[8] Nasr, H., Ahmadi, G.: The effect of two-way coupling and inter-particle collisions on turbulence modulation in a vertical channel flow, Int. J. Heat and Fluid Flow 28, 2007

[9] Sommerfeld, M., Wachen, van B., Oliemans, R.: Best Particle Guidelines, ERCOFTAC, version 20-08-2007, 2007

[10] Squires, K. D., Eaton, J. K.: Particle response and turbulence modification in isotropic turbulence, Phys. Fluids A, Vol. 2, No. 7, 1990

[11] Yamamoto, Y., Potthoff, M., Tanaka, T., Kajishima, T., Tsuji, Y.: Large Eddy Simulation of turbulent gas-particle flow in a vertical channel: effect of considering inter-particle collisions, J. Fluid Mech., vol. 442, 2001 